\title{
Interactions between metabolism and circadian clocks: reciprocal disturbances
}

\author{
Julien Delezie and Etienne Challet \\ Department of Neurobiology of Rhythms, Institute of Cellular and Integrative Neurosciences, Centre National de la Recherche \\ Scientifique, UPR3212 associated with University of Strasbourg, Strasbourg, France
}

Address for correspondence: Etienne Challet, CNRS and University of Strasbourg, Institute of Cellular and Integrative Neurosciences, 5 rue Blaise Pascal, Strasbourg, FR 67000, France. challet@inci-cnrs.unistra.fr

\begin{abstract}
Obesity is a medical condition of excess body fat, recognized as a global epidemic. Besides genetic factors, overconsumption of high-energy food and a sedentary lifestyle are major obesogenic causes. A newly identified determinant is altered circadian rhythmicity. To anticipate and adapt to daily changes in the environment, organisms have developed an endogenous circadian timing system, comprising a main circadian clock, located in the suprachiasmatic nucleus (SCN) of the hypothalamus, principally synchronized to the light-dark cycle. Secondary peripheral clocks are found in various tissues, such as the liver, pancreas, and adipose tissue. These clocks control the rhythmic patterns of myriad metabolic processes. We will review the evidence that metabolic dysfunction is associated with circadian disturbances at both central and peripheral levels and, conversely, that disruption of circadian clock functioning can lead to obesity. The roots of these reciprocal interactions will be illustrated by transcriptional crosstalk between metabolic and circadian systems. Chronotherapeutic approaches of dieting to maintain or restore a proper circadian alignment could be useful to limit the magnitude of metabolic risks.
\end{abstract}

Keywords: circadian rhythm; clock gene; metabolism; obesity; feeding

\section{Introduction}

Over recent decades, obesity has become a major health problem, while at the same time being one of the leading preventable causes of death in the world. ${ }^{1}$ Obesity is a pathological condition defined as excessive fat accumulation and is associated with major complications, such as diabetes, hypertension, and cardiovascular diseases. Besides a few genetic factors, the main contributor to obesity is an overconsumption of food that is high in calories and saturated fat. In addition to high-fat, hypercaloric diets, sedentary lifestyles have supplanted regular physical activity, resulting in a positive energy balance (more energy intake, less energy expenditure) and obesity. ${ }^{2}$

Along with these factors, timing aspects of food intake and metabolic regulation have to be taken into consideration. In living organisms, most essential biological functions show a rhythmic pattern close to 24 hours. These endogenous daily varia- tions, called circadian rhythms (from the latin: circa meaning about and dies meaning day), allow organisms to anticipate and prepare for periodic changes in the environment (e.g., light-dark cycle or food availability). The timing and duration of many physiological, metabolic, and behavioral processes are therefore dictated by a circadian timing system in coordination with the environment.

After a brief introduction of the circadian timing system, we will review the literature showing that the occurrence of obesity and/or diabetes is associated with circadian disturbances. Next, we will present laboratory studies demonstrating that direct circadian disruptions can have a broad impact on metabolism. We will also highlight the fact that irregular mealtimes and/or unusual lighting conditions are major contributors of circadian misalignment, perturbing both clock rhythmicity and metabolic homeostasis. By a reinforcing effect, circadian misalignment, resulting from inappropriate timing of food intake and/or exposure to 


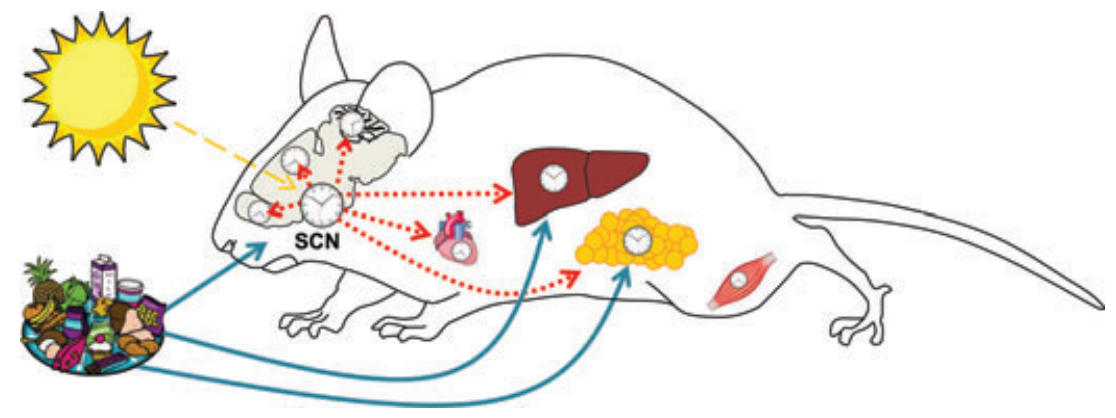

Figure 1. Organization of the circadian timing system. The master clock, located in the suprachiamatic nuclei (SCN) of the hypothalamus, adjusts the timing of many secondary clocks/oscillators in the brain and peripheral organs, in part via nervous pathways (dotted red lines). Light perceived by the retina is the most potent synchronizer of the SCN clock (dashed yellow arrow), while meal time can synchronize peripheral clocks (blue arrows).

unusual sleep/wake cycles, increases the likelihood of metabolic risk factors, and can even lead to metabolic disruptions. Finally, we will illustrate the transcriptional interactions between metabolism and the circadian system, and their importance in the development of overweight.

\section{The circadian metabolism}

\section{The circadian timing system: an overview}

In mammals, the circadian timing system is composed of several endogenous clocks, allowing biological functions to oscillate and be in phase with daily changes in the environment. The main circadian clock is located in the SCN of the anterior hypothalamus. The SCN is composed of multiple coupled cellular clocks that can generate circadian oscillations. ${ }^{3}$ For a proper phase adjustment of the circadian timing system to the external environment, SCN neurons receive direct input from the retina in order to be synchronized (i.e., reset) to the 24-h light-dark cycle. ${ }^{4}$ When light activates photoreceptors, in particular the intrinsically photosensitive retinal ganglion cells, nerve impulses are conducted directly through the retinohypothalamic tract to the SCN. ${ }^{5}$ As a result, the synchronized SCN orchestrates the rhythmicity of many aspects of metabolism, physiology, and behavior via neuronal connections and humoral factors, with a $24-\mathrm{h}$ period (Fig. 1).

The functioning of a circadian clock is dependent on a core clock mechanism involving specific genes called "clock genes." Among them period homolog 1-3 (Per1, Per2, Per3), cryptochrome 1,2 (Cry1, Cry2), circadian locomotor output cycles kaput (Clock) or its analog neuronal PAS do- main protein 2 (Npas2), brain and muscle Arntlike 1 (Bmal1), reverse viral erythroblastis oncogene products (Rev-erba and Rev-erbb), and retinoic acid-related orphan receptors (Rora, Rorb, Rorg) are essential. ${ }^{6}$ Clock genes are coexpressed (in virtually all tissues) and their products reciprocally interact at the transcriptional/translational levels to generate circadian oscillations. At least three autoregulatory feedback loops are interconnected: one positive in which CLOCK and BMAL1 dimerize to activate the E-box-mediated transcription of Per and Cry genes; one negative whereby upon reaching a critical concentration, PER and CRY proteins enter into the nucleus to inhibit the transactivation mediated by CLOCK:BMAL1, therefore inhibiting their own transcription; and an interconnecting loop, in which Rors can activate the transcription of Bmall and Npas2, whereas Rev-erbs can repress Bmal1, Clock, and Npas2, via retinoic acid-related orphan receptor response element (RORE) (Fig. 2).$^{7-10}$ This loop ensures the fine tuning of circadian rhythms. In addition, posttranslational mechanisms such as protein phosphorylation, affect stabilization, degradation, and subcellular localization of clock proteins, thus contributing to the molecular clockwork. ${ }^{11}$

\section{Circadian control of metabolic functions}

A few hypothalamic nuclei, including the paraventricular nucleus, receive direct neuronal connections from the $\mathrm{SCN}^{12,13}$ and project to peripheral tissues, such as the liver, ${ }^{14,15}$ pancreas, ${ }^{16}$ or adipose tissue $^{17}$ via autonomic output pathways. Considerable experimental work has highlighted the important role of hypothalamic nuclei in the control of feeding and energy metabolism. The ventromedial 


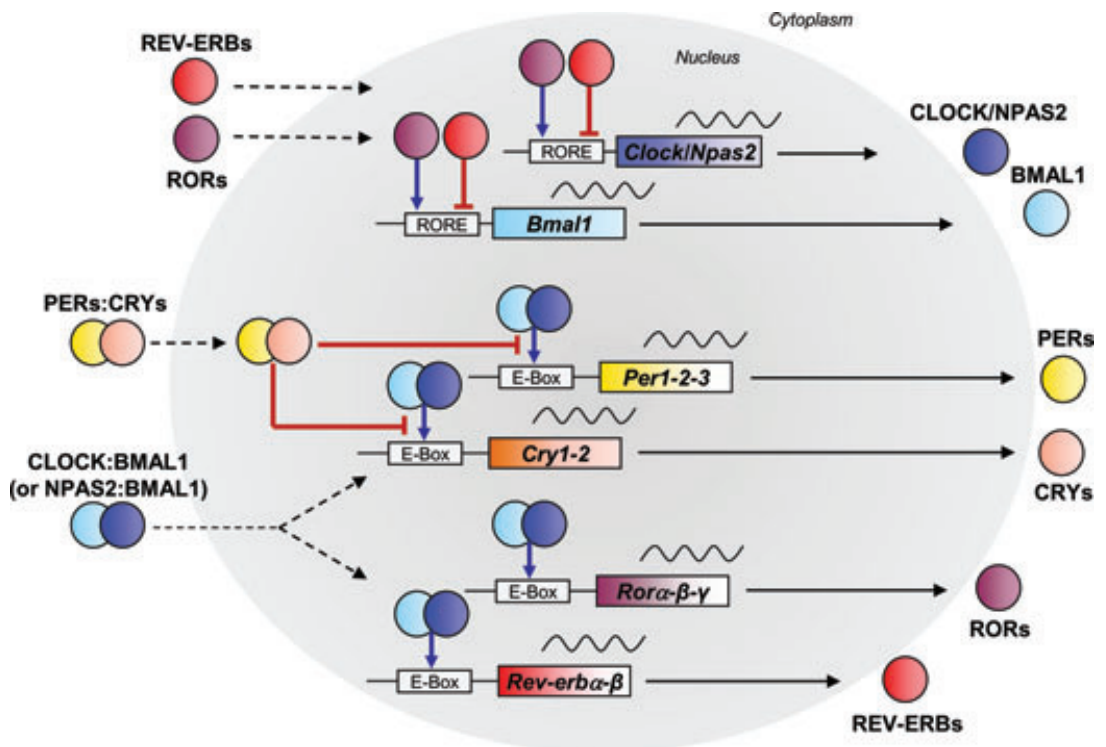

Figure 2. Simplified cellular model of the mammalian molecular clockwork. Circadian rhythms are generated by transcriptional/translational feedback regulatory loops of core clock genes. CLOCK (or NPAS2) and BMAL1 can dimerize to rhythmically transactivate genes containing a specific DNA sequence (E-box) in their promoter region. As a result, the transcriptional activity of Pers, Crys, Rors, and Rev-erbs is enhanced, and their products from translational activity are cyclically released in the cytoplasm. Then, when PERs and CRYs proteins reach a critical concentration, they form heterodimers that translocate into the nucleus to repress transcriptional activity induced by CLOCK (NPAS2):BMAL1, leading to their own repression. An additional loop involves the nuclear receptors Rev-erbs and Rors, which can translocate into the nucleus to modulate Bmal1, Clock, and Npas 2 transcription via opposite action on a RORE sequence located in their promoter.

hypothalamic nucleus, lateral hypothalamus, and arcuate nucleus (ARC) contain glucose-sensing neurons and receive nutritional information from blood-borne signals and neuronal messages from brainstem nuclei, including parabrachial nucleus and nucleus of the solitary tract (NTS). Among the key hormones that modulate feeding via hypothalamic activity, leptin synthesized by adipocytes acts on ARC, in particular, to inhibit appetite and stimulate energy expenditure. ${ }^{18}$ Insulin, released from the $\beta$ cells of the pancreas, also has anorexigenic effects on the metabolic hypothalamus. ${ }^{19}$ On the other hand, ghrelin, released by the stomach, activates neuropeptide Y/Agouti-related peptide-containing neurons in the ARC to increase appetite and decrease energy expenditure. ${ }^{20}$ An important role of the SCN is to orchestrate the internal ticking of different central and peripheral tissues. Therefore, $24-\mathrm{h}$ rhythmic patterns can be observed for a plethora of metabolic functions, such as glucose, ${ }^{21,22}$ nonesterified fatty acids (NEFA), insulin, ${ }^{22}$ and leptin levels. ${ }^{23}$
The clock genes mentioned above are not only expressed in the SCN, but also in other brain regions and peripheral tissues. ${ }^{24-26}$ In the liver, clock genes show a robust circadian pattern of expression. ${ }^{25}$ In rats, destruction of the SCN abolishes the circadian rhythm of food intake. ${ }^{27}$ SCN lesions do not abolish Per2 rhythms in peripheral tissues, but produce internal desynchrony. ${ }^{28}$ Microarray analysis of the mouse liver transcriptome showed that $9 \%$ of more than 2,000 genes studied oscillate in a circadian manner and may be under the control of the SCN. ${ }^{29}$ Thus, it is likely that the SCN modulates both behavior and metabolism by adjusting the phase of peripheral oscillators. Recent genome-wide transcriptome analyses performed in the SCN, liver, and adrenal glands of mice, revealed that around $10 \%$ of transcripts are regulated in a circadian manner. ${ }^{30-35}$ Of the 49 nuclear receptors that are key actors of metabolic regulations (see below), approximately $40 \%$ were cyclic in the liver or white adipose tissue (WAT). ${ }^{36}$ Liver posttranscriptional and translational mechanisms contribute as 
well to circadian coordination. ${ }^{37}$ While the master clock in the SCN is mainly synchronized by light, clock gene oscillations in peripheral tissues can be shifted by feeding time (Fig. 1). ${ }^{25,38,39}$ In contrast, the SCN clock is relatively impervious to the synchronizing effect of meal time, provided that the animals are exposed to a light-dark cycle and ingest enough daily energy. Under severe food restriction (i.e., caloric restriction), however, the phase of the SCN and its synchronization to light are modified. ${ }^{40}$ Expression in the SCN of receptors to metabolic hormones (i.e., insulin, ghrelin, and leptin) raises the possibility that peripheral hormonal signals can feed back to the SCN. ${ }^{41-43}$

Therefore, the whole circadian system participates in the daily variation of metabolism. Several biological approaches and genetic models have given further insight into the maintenance of metabolic homeostasis and the crosstalk between the circadian system and metabolism, as will be further discussed below.

\section{Metabolic diseases are associated with circadian disturbances}

\section{Genetic obesity and diabetes}

Monogenic causes of obesity and diabetes are relatively rare, especially in humans. For instance, earlyonset obesity has been associated with a mutation in the leptin receptor in humans. ${ }^{44}$ In rodents, however, genetic syndromes of obesity and diabetes mellitus offer models of choice to analyze circadian disturbances associated with metabolic physiopathology.

In obese Zucker rats, which carry a mutation (i.e., $\mathrm{fa}$ ) in the leptin receptor gene, daily rhythms of locomotor activity, body temperature, and feeding are phase advanced (i.e., the active phase starts in the afternoon in contrast to the nocturnal onset in $+/$ ? littermates) and their day-night amplitude is generally reduced. ${ }^{45-47}$ Increased daytime feeding is also observed in obese mice carrying the null mutation of a receptor to prostaglandin E2 (i.e., EP3 subtype). ${ }^{48}$ As a rule, the amplitude of the sleep-wake cycle and activity-rest rhythm is dampened in genetically obese rats and mice, diabetic or not, due in part to increased wake/activity during daytime and increased sleep at night. ${ }^{4-54}$

The molecular clockwork in peripheral tissues is consistently disrupted in genetically obese, diabetic or not, mice. More precisely, in obese $o b / o b$ and
$\mathrm{KK}$ mice as well as in obese and diabetic $\mathrm{KK}-\mathrm{A}^{\mathrm{Y}}$ mice, the amplitude of daily profiles of clock gene expression in the liver or white adipose tissue is generally reduced, if not barely sizeable. ${ }^{55,56}$ In the liver of obese and diabetic $d b / d b$ mice, the molecular clockwork is differentially modified according to the clock gene considered. ${ }^{51}$

Of interest, in $o b / o b$ mice, alterations in peripheral clocks occur earlier than metabolic symptoms, such as morbid obesity and hyperinsulinemia, thus suggesting that altered clockwork can play a role in obesity in addition to the specific deficiency of leptin. ${ }^{55}$ Within the central nervous system of $o b / o b$ or $\mathrm{KK}-\mathrm{A}^{\mathrm{Y}}$ mice, clock gene oscillations have been studied in the NTS. The most salient changes concern Bmall and Rev-erba, whose daily expression is upand downregulated, respectively. ${ }^{57}$ Moreover, the molecular clockwork of the SCN is not markedly affected in leptin-deficient, obese (i.e., ob/ob) mice. ${ }^{55}$ Unexpectedly in view of the severe dampening of overt rhythmicity in $d b / d b$ mice, the amplitude of SCN molecular oscillations is significantly increased in these mice compared to $d b /+$ littermates. ${ }^{51} \mathrm{Fi}$ nally, it is worth mentioning that $o b / o b$ mice show increased photic resetting of the master clock. ${ }^{54}$

\section{Diet-induced obesity}

In nature, species are not often confronted with food abundance. On the contrary, food can be rare for most animals and foraging is a key for survival (thus contributing to energy expenditure). Consequently, obese animals in the wild are uncommon, except for some species in which overweighting is important to support long periods of fasting (e.g., penguins, bears), and those species living in proximity to humans and consuming greasy food that is discarded by people. Most laboratory-based research on obesity is carried out in rodents. ${ }^{58}$ Control animals usually have an ad libitum access to a normocaloric chow diet. Despite this free access, most rodents regulate their consumption and the time when they eat and do not become obese.

Obesity can be induced by feeding rodents on an ad libitum basis with a high-fat diet (HFD, more than $50 \%$ of metabolizable energy derived from fat). Both the amount and dietary fat type alter body mass and composition. ${ }^{59}$ Short-term high-fat feeding reduces circadian variations of leptin levels in rats ${ }^{60}$ and humans, ${ }^{61}$ thus suggesting that dampened circulating leptin could contribute to the 
development of obesity. In mice, high-fat feeding attenuates the daily pattern of food intake, with a higher consumption during the day and a concomitant decrease during the active period, before significant mass gain. In WAT of mice fed with HDF, expression of metabolic genes can be downregulated at night, such as sterol regulatory element-binding protein 1c $(S r e b p-1 c)$ or fatty-acid binding protein 4 (Fabp4), while expression of others is barely affected, like acetyl-CoA carboxylase $(A c c)$ and fatty acid synthase (Fas). Other transcriptional changes are detected in the liver of these mice: expression of Acc, Fas, and Fabp1 is upregulated, while the nighttime peak of Srebp-1c is phase-advanced to the afternoon. Moreover, changes in the concentration and temporal pattern of expression of glucose, insulin, leptin, and NEFA are also observed. ${ }^{62}$ Highfat feeding also leads to difficulties in maintaining wakefulness during the active period and increases nonrapid eye movement sleep in mice ${ }^{63}$ as well as postprandial sleepiness in humans, ${ }^{64}$ suggesting that the metabolic state affects neural structures regulating sleep. Equally interesting, HFD seems to have a direct effect on the main circadian clock. In mice, HFD lengthens the free-running period ${ }^{62}$ and disrupts photic synchronization of the SCN to light, as shown by slower re-entrainment to shifted lightdark cycle and reduction in light-induced phase shifts. ${ }^{65}$ There is also a clear change in neuropeptide expression in the mediobasal hypothalamus, despite no major modification of the core clock machinery in that region. ${ }^{62}$ In the brainstem, more precisely in the NTS, mice fed with HFD display altered daily patterns of clock gene expression, including downregulated Rev-erba, and upregulated Bmall and Clock mRNA levels. ${ }^{57}$ Taken together, these results suggest that central dysfunctions may contribute to the development of obesity.

Oscillations of clock genes have been found in human WAT, ${ }^{66}$ but no difference has been detected in the characteristics of these oscillations between lean, overweight, and diabetic individuals. ${ }^{67}$ By contrast, the circadian timing of peripheral tissues is markedly modified by fat overload in rodents. HFD alters the diurnal variation in glucose tolerance, and insulin sensitivity by influencing clock functioning. ${ }^{68}$ In the liver, changes are seen in the level as well as the rhythmic pattern of major components of lipid homeostasis and adiponectin metabolic pathway, with parallel modifications in clock gene ex- pression. ${ }^{62,69}$ In HFD-fed animals, expression of metabolic actors is asynchronous in liver and adipose tissue, suggesting the importance of temporal coordination among metabolic tissues. Furthermore, HFD also attenuates the amplitude of Clock, Bmal1, and Per2 in adipose tissue. ${ }^{62}$ Hence, altered circadian clock function within adipose tissue may promote excess fat storage (especially intraabdominal fat). However, it is important to note that HFD-induced changes in clock gene expression could be gender specific, since a recent study in female mice did not obtain the same results. Indeed, eight weeks of HFD had only mild effects on clock gene profiles in the liver and adipose tissue, in spite of the presence of hyperlipidemia, hyperglycemia, and overweight. ${ }^{70}$ Moreover, the time and duration of feeding are also important. Four or eight weeks of HFD failed to alter peripheral clocks, whereas 11 weeks did. ${ }^{70,71}$ The differences between strains, gender, starting age of HFD feeding, and its duration, add a level of complexity to the comprehension of the risk factors that trigger obesity in rodents.

In summary, impairment of clock gene oscillations and metabolic pathways may explain the altered coordination of metabolic functions and clock-controlled output signaling, contributing to obesity and associated disorders (e.g., diabetes, sleep disturbances). Recent evidence also demonstrates that disruption of the circadian timing system solely, has various consequences on metabolism.

\section{Circadian disruption is associated with metabolic dysfunctions}

\section{Altered endogenous clockwork}

As aforementioned, clock genes are expressed in virtually all tissues and circadian clocks participate in the daily regulation of metabolic functions such as glucose and lipid metabolism. ${ }^{22,68}$

One of the first clock genes studied was Clock. Homozygous C57BL/6J Clock mutant mice are hyperphagic, show a dampened feeding pattern, with increased food intake during the rest period, and attenuated energy expenditure at night, thus contributing to fat excess. Clock mutant mice display severe metabolic alterations, including hypercholesteronemia, hypertriglyceridemia, hepatic steatosis, and hyperglycemia. In addition, Clock mutation leads to day-night changes in the expression of 
hypothalamic neuropeptides, like CART (Cocaine and amphetamine regulated transcript) and orexin mRNA, which play a role in central circuits controlling feeding and arousal. ${ }^{72}$ Furthermore, Clock mutation induces profound changes in glucose homeostasis, such as increased insulin sensitivity and altered gluconeogenesis. ${ }^{68}$ Of interest, in the liver, Clock has been shown to directly drive the expression of glycogen synthase 2 gene via E-boxes. ${ }^{73}$ In Clock mutant mice generated on a CBA/6CaH background (which synthesize melatonin contrary to the C57BL/6J strain), impairments in glucose tolerance, insulin secretion, liver Pepck mRNA expression, and increased insulin sensitivity are also observed, although these mice do not become spontaneously obese unlike C57BL/6J Clock mutants. ${ }^{74}$ Moreover, Clock (Jcl:ICR) mutant mice display levels of triglycerides and NEFA lower than control mice, and cholesterol and glucose levels do not differ. Due to reduced lipid absorption and hepatic lipogenesis, these mice challenged with HFD show reduced body mass elevation, leptin, and insulin levels. ${ }^{75,76}$ In spite of these discrepancies, due notably to strain-related differences, mutation of the Clock gene clearly has an impact on lipid metabolism, since Clock has been shown to participate in liver cholesterol accumulation ${ }^{77}$ and to amplify obesity in $o b / o b$ mice. $^{78}$

Bmal1, a close partner of Clock, has been shown to participate in adipocyte differentiation and lipogenesis. ${ }^{79}$ Sensitivity to exogenous insulin is increased by deletion of Bmal1, while gluconeogenesis is suppressed. ${ }^{68}$ Liver-specific deletion of Bmal1 in mice nicely confirmed its strong involvement in glucose metabolism. $L$-Bmal1 ${ }^{-/-}$mice have impaired expression of clock-related genes involved in hepatic glucose regulation such as genes encoding glucose transporter 2, glucokinase, or pyruvate kinase. $L-\mathrm{Bmal1}^{-/-}$mice were hypoglycemic during the resting period, were more glucose tolerant, had normal insulin sensitivity and production, and normal total body fat content compared to wild-type mice. ${ }^{80}$ Altogether, these results demonstrate that Clock and Bmal1 modulate hepatic function to regulate glucose and fatty acid homeostasis. Interestingly, two Bmal1 haplotypes have been associated with diabetes in humans ${ }^{81}$ as well as Clock polymorphisms and related haplotypes that may play a role in the development of obesity. ${ }^{82,83}$
Recent elegant studies have explored the role of Clock and Bmal1 in the pancreatic islets, demonstrating that the pancreas harbors a functional circadian oscillator. $^{84,85}$ They showed that pancreasspecific Bmal1 mutant mice have higher blood glucose levels during the whole 24-h cycle, impaired glucose tolerance, and reduced insulin secretion. Moreover, pancreatic islets of these mutant mice have altered development and produced less insulin, suggesting that intact clock functioning in the pancreas is essential for normal insulin secretion. ${ }^{85}$ This study establishes clearly that circadian components can regulate local metabolism.

Mutation of the core clock gene Per2 can also lead to abnormal conditions. In Per2 ${ }^{-/-}$(Per2tm1Brd) mice, daily corticosterone rhythm is markedly attenuated. Of interest, when fed with HFD, these mice eat more during the rest period compared to wild-type mice and develop obesity. ${ }^{86}$ Moreover, the rhythmic pattern in Per2 $2^{-/-}$mice of alphamelanocyte-stimulating hormone ( $\alpha-\mathrm{MSH})$, a powerful appetite suppressing peptide, is disrupted and peripheral injection of $\alpha-\mathrm{MSH}$ induces weight loss. ${ }^{86}$ Interestingly, the circadian rhythm of Per2 was dampened in the mediobasal hypothalamus of Clock mutant mice that are obese. ${ }^{72}$ In mice in which Per2 is fully ablated, lipid metabolism was altered with decreases in both plasma NEFA and total triacylglycerol (TAG), and TAG contents in the WAT. ${ }^{87}$ In Per2 ${ }^{-/-}$mice, and to a lesser extent in $\mathrm{Per}^{-/-}$mice, glucose tolerance was increased (i.e., improved) compared to wild-type animals. ${ }^{88}$ By contrast, in Per1 ${ }^{-/-}$and $P e r 2^{-/-}$double-mutant mice (129/sv background), glucose tolerance and insulin sensitivity were both attenuated. ${ }^{80}$ Triplemutant mice for Per1-Per2-Per3 gain more body mass on HFD than wild-type mice, similar to Per3 single-mutant mice, thus indicating that the Per3 mutation alone accounts for the obese phenotype. ${ }^{89}$

Along with core clock components, secondary actors of circadian oscillations take part in metabolic functions. Rora, activator of Bmall transcription, has been shown to participate in the regulation of apolipoprotein C-III (Apo C-III) gene involved in triglyceride metabolism, ${ }^{90}$ and lipid homeostasis in skeletal muscle. ${ }^{91}$ Rev-erba, repressor of Bmall, is involved in adipocyte differentiation ${ }^{92,93}$ and rhythmic bile and lipid homeostasis. ${ }^{94}$ Rev-erba ${ }^{-/-}$mice displayed elevated serum Apo C-III and triglycerides levels. ${ }^{95}$ Moreover, although the deletion of Rev-erba 
alone has only a minor effect on hepatic glucose regulation, ${ }^{94}$ the alterations are more pronounced with concomitant mutation of Per $2 .{ }^{96}$ Indeed, the expression of key enzymes of glucose metabolism, such as glucose-6-phosphatase or glucokinase, was more affected in the liver of Rev-erba ${ }^{-/-} /$Per 2 mutant mice.

From all these results, it is tempting to think that studying the effects of clock gene disruption could shed light on the incapacity of physiological control mechanisms to cope with adverse consequences, for instance of overweight. However, the effects of a mutation in the Nocturnin gene, which encodes a circadian deadenylase important for posttranscriptional regulation of circadian-related genes, ${ }^{97}$ do not fit with this thought. Nocturnin knockout mice exhibit no change in circadian rhythm of locomotor activity and liver clock gene expression, although they have altered expression of metabolic genes, reduced lipid absorption, and are resistant to diet-induced obesity. This study indicates that circadian metabolism can be disrupted without noteworthy modification in core clock functioning while a specific subset of circadian downstream targets involved in lipid metabolism is affected..$^{98}$ Ongoing research into the complex crosstalk between components of the circadian system and metabolism may lead to prevention and improve treatment of metabolic disorders in the future.

\section{Altered rhythmic "environment"}

There are two main external causes of disruption in circadian rhythmicity. The first one concerns chronic changes in timing of light-dark cycles, such as chronic jet lag. The second way relates to behavioral activity (e.g., physical activity, feeding) occurring during the usual resting period (nighttime in humans, daytime in nocturnal rodents) with long-term shifts, such as during night work (also called permanent shift work), or short-term shifts, like rotating shift work. As detailed below, these desynchronizing situations have deleterious consequences on circadian organization and metabolic health.

Housing mice in light-dark cycles that are too short (i.e., $20 \mathrm{~h}$ ) for enabling daily synchronization of their master clock leads to larger body mass gain and increased insulin/glucose ratio, indicative in the fasted state of insulin resistance. ${ }^{99}$ The same paradigm has also been shown to aggravate car- diovascular disease in mice. ${ }^{100}$ In human subjects exposed to controlled 28-h sleep-wake cycles under dim light (so-called "forced desynchronization" protocol), circadian misalignment impairs glucose tolerance and reduces sensitivity to insulin. ${ }^{101} \mathrm{Ad}-$ ditionally, constant exposure of mice to bright or dim light leads to increased body mass and reduced glucose tolerance compared to mice housed under a regular light-dark cycle. ${ }^{102}$

Repeated weekly shifts of 24-h light-dark cycle in rats fed with regular chow diet lead to chronic desynchronization and trigger higher body mass gain $^{103}$ as well as impaired insulin regulation. ${ }^{104}$ Interestingly, expression of FABP4 is upregulated in WAT of rats exposed to repeated light-dark shifts. ${ }^{105}$ A number of epidemiological studies in different countries have highlighted increased metabolic risks for cardio-metabolic syndrome, including increased disturbances in lipid metabolism (e.g., high levels of cholesterol and triglycerides), ${ }^{106-110}$ glucose metabolism (e.g., high fasting insulin and glucose), ${ }^{108,111}$ increased concentration of leucocytes, reflecting systemic inflammation, ${ }^{108}$ and hypertension. ${ }^{106,110}$

Chronic sleep disturbance or sleep restriction under a regular light-dark cycle alters glucose homeostasis in rats. ${ }^{112}$ In humans, recurrent sleep debt is a newly identified risk factor for obesity and diabetes, ${ }^{113,114}$ indicating that it is likely an aggravating factor for metabolic disturbances in night workers.

Being nocturnal animals, laboratory rats and mice exposed to a light-dark cycle consume most of their daily food during the night period. In rodents, daytime feeding is a potent synchronizer of peripheral clocks. ${ }^{25,38,39,115}$ Access to food restricted to a few hours during the light phase usually leads to mild body mass loss or no change at all. ${ }^{38,116,117}$ In more rare cases, food-restricted rats with chow diet available only for a few hours can increase their body mass. ${ }^{118}$ A clear contribution of circadian timing of food intake in body mass gain has been shown in Zucker rats (Fig. 3). ${ }^{46}$ As mentioned above, $f a / f a$ rats ingest a larger proportion of food during the usual resting phase (i.e., light phase) than control animals. This study was the first to demonstrate that by limiting food access to the normal period of activity and feeding (i.e., nighttime in nocturnal rats), Zucker rats gained less body mass compared to free-fed animals, in spite of similar amounts of food intake between both groups. ${ }^{46}$ More recent 


\section{Zucker rats}
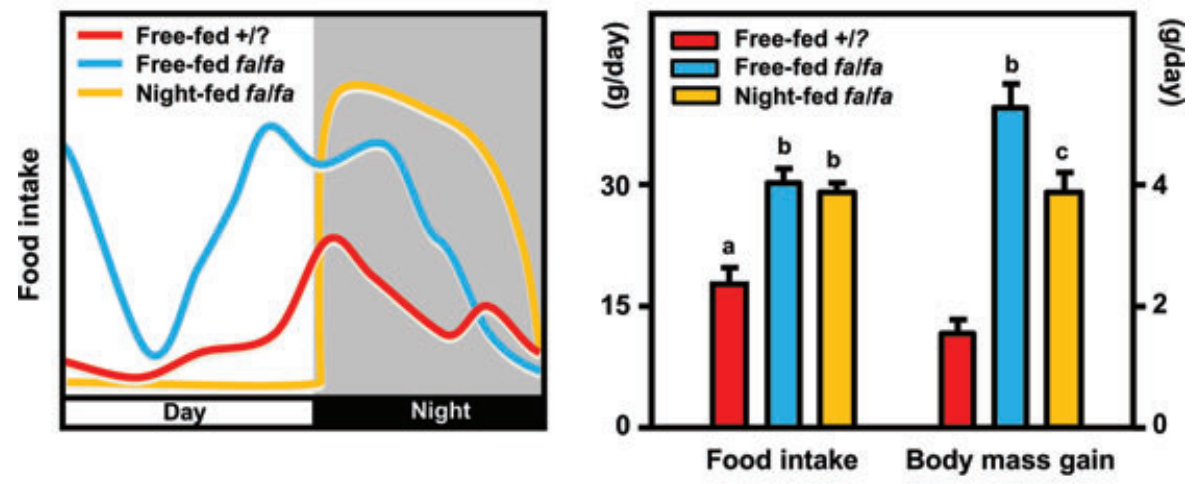

C57BL mice
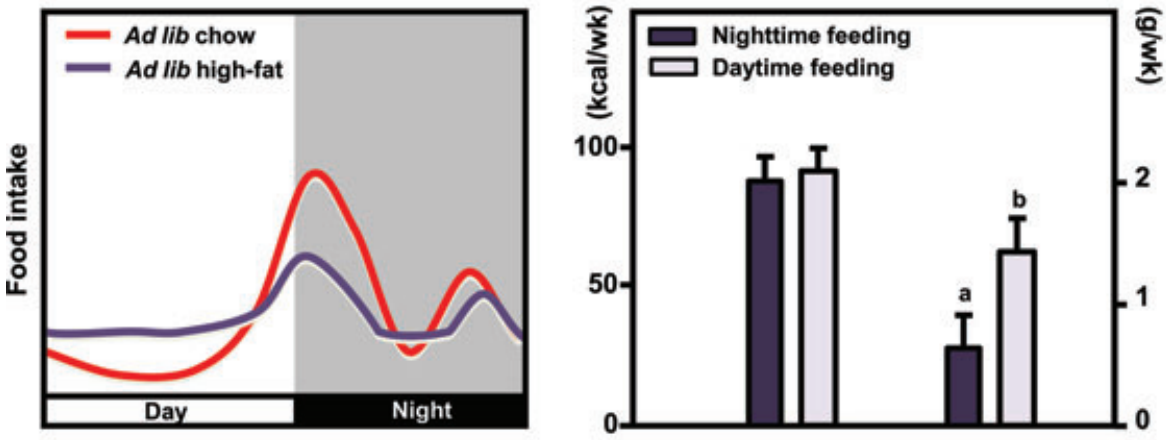

High-fat intake Body mass gain

Figure 3. Importance of feeding time in genetic or diet-induced obesity. In Zucker rats (upper panels), obese individuals (i.e., $f a / f a$; blue lines and bars) eat more during daytime than control littermates (+/?; red lines and bars). When food access is limited to nighttime (orange lines and bars), $f a / f a$ rats gain less body mass in spite of similar whole energy intake compared to free-fed individuals (modified with permission from Ref. 46). In C57BL6 mice (lower panels), free access to a high-fat diet is associated with an increase in daytime feeding (purple line), while chow feeding is mostly nocturnal (red line). If high-fat feeding is restricted to daytime or nighttime hours (light and dark purple bars, respectively), body mass gain is larger in the former, despite comparable energy intake (drawn from data in Refs. 62 and 118).

observations confirm nicely the metabolic consequences of unusual timing of feeding. Mice fed ad libitum with HFD display a rapid increase in daytime feeding that takes place weeks before the obese phenotype is detectable. ${ }^{62}$ Furthermore, when HFD was restricted to the light phase, mice gained more body mass than those on the same diet but with access limited to the dark phase (Fig. 3). ${ }^{119}$

Another paradigm used to awaken rodents during their resting period (i.e., daytime) is forced activity that leads to internal desynchronization. ${ }^{120}$ Keeping food intake to the normal feeding period (i.e., night) in spite of diurnal forced activity prevents body mass and metabolic changes. ${ }^{121}$ Considering that exposure to bright light at night is an aggravating factor for the occurrence of pathologies in human night workers, ${ }^{122}$ it may be clinically relevant to develop shift-work models in day-active animals.

In humans, night-eating syndrome (NES) is characterized by a delayed pattern of calorie intake (i.e., lack of appetite in the morning and evening or nocturnal hyperphagia). In contrast to this delayed meal timing, the daily rhythm of plasma ghrelin is largely phase advanced, while the rhythm in plasma glucose is phase opposed in patients with NES compared to those in control subjects. ${ }^{123}$ Elevated nocturnal, but not "evening," feeding has been correlated with an increased risk of overweight in some, ${ }^{124,125}$ but not all epidemiological studies. $^{126}$ 


\section{Transcriptional networks connecting molecular clockwork and metabolic pathways}

As aforementioned, a set of clock genes is responsible for the generation of circadian oscillations. From genetic strategies allowing modifications of the genome in mice, it has been discussed above that most, if not all, clock gene deletions lead to a broad range of metabolic diseases. In addition, we have introduced the effects of unhealthy food and incorrect timing of food intake on metabolic regulatory centers and the core clock machinery. In this last section, we will give an overview of the emergent understanding of the communication within the circadian clock circuitry. We will illustrate the different molecular pathways by which clock-related nuclear receptors are involved in metabolism and the effects of nutrient "sensors" on core clock components (Fig. 4).

\section{The nuclear receptors' dynamic network}

Transcriptomal analyses have demonstrated that a large number of nuclear receptors are expressed in a circadian manner. ${ }^{36}$ Among them, Rors, Rev-erbs, and peroxisome proliferator-activated receptor (Ppars) genes appear to be pivotal players at the interface between the circadian system and metabolism. ${ }^{127}$

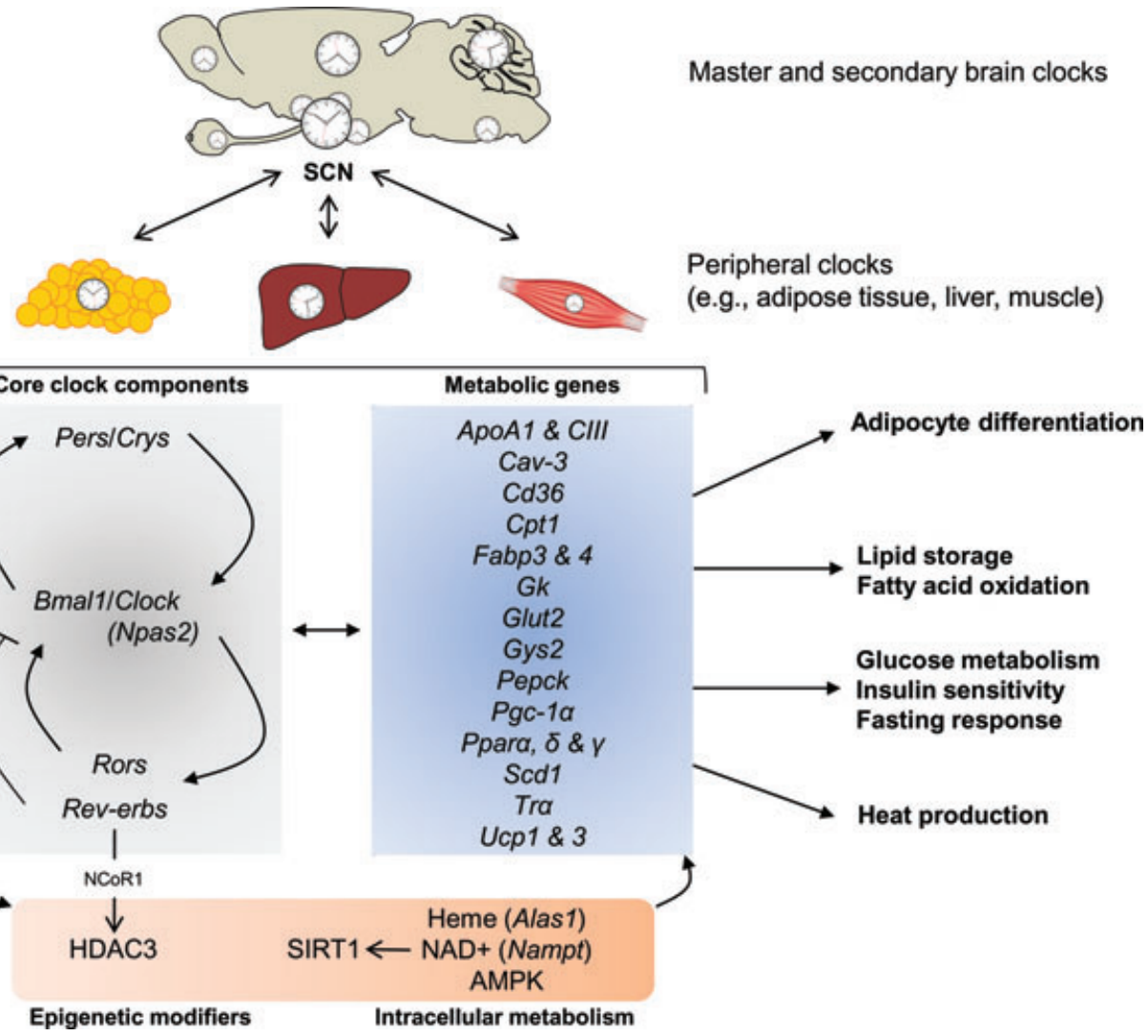

Figure 4. A schematic illustration of the cross-talk between circadian components and metabolic regulators. The master clock housed in the SCN is connected to several brain and peripheral clocks to orchestrate rhythmic activities. All clocks share a common molecular mechanism in which the clock genes are coexpressed, and the generation of circadian oscillations is made from transcriptional/translational interactions (represented in the gray box; for more details, see Fig. 2). Clock genes can influence, directly or indirectly, the rhythmic expression of many metabolic genes (listed in the blue box). Clock components can also control rate-limiting enzymes in the $\mathrm{NAD}^{+}$salvage pathway (i.e., Nampt) and heme biosynthesis (i.e., Alas1). Intracellular metabolism through the action of SIRT1, AMPK, and heme can impinge both metabolic genes and core clock machinery. In addition, epigenetic modifiers, such as HDAC3 recruited by REV-ERB $\alpha$ via NCoR1, modulate clock and metabolic gene transcription (orange box). 
As introduced above, RORs and REV-ERBs compete for binding RORE in clock gene promoters. ${ }^{7-10}$ Interestingly, Rev-erba can repress its own transcription on a functional REV-ERB $\alpha$ binding site (RevDR2) located in its promoter. ${ }^{128}$ ROR $\alpha$ being able to bind to this site participates in the control of Rev-erba transcription. ${ }^{129}$ Hence, genes containing either RORE or RevDR2 in their promoter are activated by ROR $\alpha$ and repressed by REV-ERB $\alpha$. This highlights the occurrence of dynamic interactions within the core clock machinery. In skeletal muscle, Rora directly regulates the mouse caveolin-3 (Cav-3) and carnitine palmitoyltransferase-1 (Cpt1) genes, involved in fatty acid metabolism. ${ }^{91}$ Rora also enhances activity of the human Apo C-III gene promoter $^{90}$ and binds to the rodent apolipoprotein A-I (ApoA1) RORE. ${ }^{130}$ Additional evidence for a role of Rora in metabolism is provided by the fact that cholesterol is a putative ligand of ROR $\alpha .{ }^{131}$

Numerous studies have shown that REV-ERBs also influence lipid and energy homeostasis. The expression of a dominant negative version of Rev$e r b b$ in transfected cell lines attenuates expression of genes involved in lipid metabolism, such as Fabp34 , cluster of differentiation 36 (Cd36), stearoylcoenzyme A desaturase 1 ( $S c d 1$ ), and cellular energy balance (e.g., uncoupling protein 3, Ucp3). Similarly to Rora, Rev-erba can bind directly to the human Apo C-III promoter ${ }^{95}$ and the rat Apoa-I promoter RORE. ${ }^{132}$ For the repressive action on Bmal1 transcription, Rev-erba has been shown to recruit the nuclear receptor corepressor (NCoR)/histone deacetylase 3 (HDAC3) complex. ${ }^{133}$ Of interest, genetic disruption of NCoR1-HDAC3 interaction in mice induces changes in Bmal1 expression and circadian behavior with concomitant effects on metabolism (e.g., increased insulin sensitivity, altered expression of metabolic genes). Surprisingly, loss of a functional NCoR1-HDAC3 complex also protects mice from diet-induced obesity. ${ }^{134}$ On the other hand, a recent study has explored the role of the circadian genomic recruitment of HDAC3 in the mouse liver. They showed that REV-ERB $\alpha$ controls the circadian expression of lipid metabolism genes by recruiting the repressive chromatin modifier HDAC3 (and NCoR) to the genome during the light period, therefore preventing lipogenesis at a time when animals are resting. In addition, deletion of either Hdac3 or Rev-erba results in hepatic steatosis indicating steady lipogenesis. ${ }^{135}$ These findings demonstrate that the circadian control of epigenetic modifiers by clock components is critical for normal metabolic processing. Additional evidence of the involvement of Rev-erbs in metabolism is provided by studies on heme, which has diverse biological functions, including oxygen sensing, cell respiration, and metabolism. ${ }^{136}$ Heme, whose expression occurs in a circadian manner, ${ }^{137}$ can bind to both REV-ERBs. ${ }^{138}$ Heme binding to REV-ERBs facilitates recruitment of the nuclear corepressor NCoR. ${ }^{133,138}$ The ratelimiting enzyme in heme biosynthesis, aminolevulinate synthase 1 (Alas 1 ), is directly controlled by the clock. ${ }^{139}$ Heme biosynthesis is also influenced by the nutritional status through the regulation of Alas 1 by the PPAR $\gamma$ coactivator $1 \alpha(\mathrm{PGC}-1 \alpha) .{ }^{140}$ This nuclear receptor coactivator is induced by fasting ${ }^{140}$ and participates in a variety of metabolic pathways such as glucose and lipid regulation. ${ }^{141}$ Moreover, both PGC- $1 \alpha$ and heme are able to modulate the expression of circadian genes. ${ }^{137,142,143}$ These results illustrate how REV-ERBs can sense dynamic metabolic changes and transmit them to the core clock machinery.

PPARs are members of the nuclear hormone receptor superfamily of ligand-activated transcription factors. The three PPARs $(\alpha, \delta, \gamma)$ have been shown to regulate carbohydrate, lipid, lipoprotein, and energy metabolism. ${ }^{144,145}$ Ppara is rhythmically expressed in tissues with high fatty acid catabolism rates such as the liver and adipose tissue. Pparg is rhythmically expressed in liver and WAT, while Ppard expression oscillates in liver and brown adipose tissue (BAT) ${ }^{36}$ PPAR $\alpha$ is likely to play a role in connecting circadian physiology to metabolism. Ppar $\alpha$-deficient mice $\left(\right.$ Ppara $\left.^{-/-}\right)$have normal rhythmic behavior and clock gene expression in the SCN, but PPAR $\alpha$ deficiency alters Bmal1 and Per3 expression in the liver. Moreover, Ppara deficiency leads to disrupted phase resetting of Bmall when food is provided during the light period, indicating that PPAR $\alpha$ may control Bmall. Indeed, PPAR $\alpha$ directly regulates hepatic Bmall expression through a response element located in its promoter. ${ }^{146}$ Besides, it has been shown that Ppara is a clock-controlled gene $e^{147}$ and daytime feeding inverts its circadian expression pattern in the liver. ${ }^{146}$ Interestingly, PPAR $\alpha$ is also connected to the nuclear receptor Rev-erba because the PPAR $\alpha$ agonists (fibrates) induce liver expression of Reverba. In addition, PPAR $\alpha$ (as well as PPAR $\gamma$ ) can 
directly transactivate Rev-erba via RevDR2 located in its promoter. ${ }^{92,132,148}$ Clearly, all these data demonstrate that the metabolic sensor PPAR $\alpha$ may play a prominent role in circadian functioning. Other results indicate that PER2 has the capacity to recruit PPAR $\alpha$ or REV-ERB $\alpha$ for the modulation of Bmal1 expression, ${ }^{96}$ again showing intimate interactions between clock and metabolic components in the clock circuitry.

PPAR $\gamma$ and PPAR $\delta$ are both involved in energy homeostasis. A recent study reveals that PER2 can interact directly with PPAR $\gamma$ to repress its transcriptional activity. Ablation of Per2 in cell culture increased induction of adipogenic genes, while deletion of Per2 in mice results in slight changes in circadian expression of PPAR $\gamma$ target genes in WAT. ${ }^{87}$ These results demonstrate that the control of PPAR $\gamma$ by a circadian actor is essential for normal lipid metabolism. Interestingly, PGC-1 (PPAR $\gamma$ coactivator 1 , as introduced above), which is involved in thermogenesis and associated metabolic responses, ${ }^{142,149}$ is rhythmically expressed in phase with Pparg, Ppard, and thermogenic genes, such as Ucp1 and thyroid receptor $\alpha($ Tra $)$, in BAT. ${ }^{36}$ Furthermore, PGC- 1 can specifically bind PPAR $\gamma,{ }^{149}$ whereas the latter can also interact with the Ucp1 promoter. ${ }^{150}$ All these data suggest that PPARs could transmit (diurnal) changes in heat dissipation to the circadian system.

These data suggest that nuclear receptors are controlling an amazing array of metabolic functions and are capable of communicating the body's metabolic state to the core clock machinery. Incidentally, other nuclear receptor subgroups also participate in the interplay between metabolic and circadian physiology (e.g., retinoid X receptor, liver X receptor, and estrogen-related receptor). ${ }^{36,127,151}$

\section{Nutrient sensors interact with clock components}

The tight connection between the circadian timing system and metabolism is also underscored by the role of nutrient sensors. Clock adaptation to metabolic reactions is likely to be achieved also by direct input of nutrient sensing regulators to the intrinsic clock machinery. However, we are aware that the subdivision between nuclear receptors presented above and metabolic sensors that we will introduce in this section may appear rather arbitrary.
Recent evidence has demonstrated that local changes in cellular energy, such as redox reactions (portmanteau for reduction-oxidation), can influence the circadian expression of core clock genes and clock-related nuclear receptors. Nicotinamide adenine dinucleotide $\left(\mathrm{NAD}^{+}\right)$and its reduced form NADH are coenzymes found in all living cells. $\mathrm{NAD}^{+}$also exists in a phosphorylated form $\mathrm{NADP}^{+}$and can be reduced to NADPH. $\mathrm{NAD}(\mathrm{P})^{+}$and $\mathrm{NAD}(\mathrm{P}) \mathrm{H}$ are involved in cellular redox reactions. Interestingly, CLOCK:BMAL1 and NPAS2:BMAL1 heterodimers can sense intracellular redox status. The DNA-binding activities of these dimers are influenced by both reduced and oxidized forms of $\mathrm{NAD}(\mathrm{H})$ and $\mathrm{NADP}(\mathrm{H})$ in an opposing manner. The reduced forms $(\mathrm{NADH}$ and $\mathrm{NADPH}$ ) activate DNA binding of CLOCK:BMAL1 and NPAS2:BMAL1, whereas the oxidized forms $\left(\mathrm{NAD}^{+}\right.$and $\left.\mathrm{NADP}^{+}\right)$inhibit it, consistent with a role as redox sensors. ${ }^{152}$ These results raise the possibility that cell energy metabolism can influence circadian rhythmicity. For example, these clock protein sensors might participate in the rhythm imposed by the alternation of fasting and feeding. Indeed, NAD:NADH ratio is modified under fasting and feeding conditions since starvation lowers NADP:NADPH (i.e., shift toward a reduced state) in both rat liver mitochondria and cytoplasm. ${ }^{153}$ Thus, for feeding synchronization, it is plausible that the cellular redox status could transmit changes in energy metabolism directly to the clock architecture. In this context, it is tempting to think that irregular mealtimes could also contribute to circadian misalignment by subtle perturbations of local circadian oscillator functioning.

Sirtuin 1 (SIRT1), another energy sensor, has recently been found to link circadian to metabolic physiology. SIRT1 is a NAD-dependent histone deacetylase that contributes to epigenetic gene silencing and a plethora of biological processes ranging from gluconeogenesis, insulin secretion and sensitivity, lipid regulation, mitochondrial activity, thermogenesis, adipogenesis and adipocyte differentiation, and apoptosis. SIRT1 also plays a crucial role in the caloric restriction-dependent life span extension. ${ }^{154-156}$ SIRT1, whose expression occurs in a circadian manner, has been shown to influence circadian transcription of several clock genes (e.g., Per2, Cry1, Bmal1, and Rora). ${ }^{157}$ Moreover, SIRT1, can directly bind CLOCK:BMAL1 heterodimers and 
promote deacetylation of PER2, ${ }^{157}$ BMAL1, and histone H3. ${ }^{158}$ Interestingly, SIRT1 can also affect expression of metabolic regulatory transcription factors. It can interact with and deacetylate PGC- $1 \alpha$ to control the expression of gluconeogenic and glycolytic genes. ${ }^{159}$ In addition, hepatic SIRT1 is able to modulate the expression of PPAR $\alpha .{ }^{160}$ Importantly, SIRT1 can also repress the fat regulator PPAR $\gamma$ and it is activated during fasting to promote fat mobilization in WAT, again demonstrating its clear implication in lipid homeostasis. ${ }^{161}$ Furthermore, SIRT1 has been shown to be decreased in adipose tissue endothelial cells from obese human subjects. ${ }^{162}$ Of interest, since fat accumulation is associated with several adverse complications, such as diabetes and hypertension, caloric restriction (and consequent fat depletion) that has multiple biological and lifeextending benefits ${ }^{163}$ may be used to prevent or treat metabolic disruptions.

In addition to $\mathrm{NAD}(\mathrm{P})^{+}$and SIRT1, AMPactivated protein kinase (AMPK) is a further important nutrient sensor. AMPK is sensitive to fluctuations in the cellular AMP:ATP ratio and can be activated by various factors such as exercise, glucose deprivation, or leptin treatment. The functions of AMPK cover the whole-body energy balance (e.g., food intake, body mass, lipid and glucose homeostasis, cholesterol and triglyceride synthesis, energy expenditure). ${ }^{164}$ In mouse skeletal muscle and cultured myotubes, AMPK has been demonstrated to regulate genes involved in energy metabolism by acting in coordination with SIRT1. Interestingly, AMPK activation increases $\mathrm{NAD}^{+}$levels, which in turn enhance SIRT1 activity, resulting in the deacetylation and activation of the downstream SIRT1 target PGC- $1 \alpha .{ }^{165}$ Deletion of the AMPK 33 subunit in mice leads to impaired expression profiles of clock genes in skeletal muscle in response to the AMPK activator AICAR (5-amino-4-imidazole-carboxamide riboside), and attenuated daily variations of the respiratory exchange ratio. ${ }^{166}$ AMPK also has direct actions on the clock machinery. AMPK phosphorylates and destabilizes CRY $1 .{ }^{167}$ Casein kinase I $\varepsilon$, an important regulator of PER proteins stability, is also phosphorylated by AMPK that induces subsequent degradation of PER2 and phase shifts of peripheral oscillators. ${ }^{168}$ Recently, it has been demonstrated that mice deficient for either AMPK $\alpha 1$ or $\mathrm{AMPK} \alpha 2$ have altered circadian feeding behavior and free- running periods. ${ }^{169}$ Surprisingly, the rhythmic gene expression of leptin, PGC- $1 \alpha$ and nicotinamide phosphoryl-transferase (NAMPT), a rate-limiting enzyme in the $\mathrm{NAD}^{+}$salvage pathway regulated by CLOCK:BMAL1, ${ }^{170,171}$ was abolished in AMPKdeficient mice. ${ }^{169}$ This study reveals that AMPK is to some extent involved in the cycling of the NAMPT-(NAD $\left.{ }^{+}\right)$-SIRT1-PGC- $1 \alpha$ pathway. In addition, prolonged activation of SIRT1 by its agonist SRT1720 causes an indirect activation of AMPK, and both SIRT1 and AMPK respond to low-energy levels. ${ }^{164,172}$ Therefore, SIRT1 and AMPK may have overlapping functions to ensure the fine tuning of metabolic and clock regulations. Consequently, if a drug exerts effects on these actors, it is advisable to also explore its influence on the core clock machinery.

Finally, it is important to mention that the mammalian molecular circadian clockwork (based on transcriptional-translational events) overviewed in this section is not a unique cellular clock model. Indeed, circadian redox rhythms of peroxiredoxins, which are antioxidant enzymes, can occur independently of transcription, thus defining a metabolic nontranscriptional oscillator. ${ }^{173}$

\section{Conclusion}

At the molecular/cellular levels, the recent findings cited above highlight multiple transcriptional crossroads between circadian and metabolic pathways in organs involved in metabolism, namely liver, adipose tissue, pancreas, and muscle. Nuclear receptors such as Rors and Rev-erbs are well situated to receive metabolic signals and integrate them into the core clock architecture. In addition, we show that fluctuations in cellular metabolism can directly influence the transcriptional activity of core clock components such as Cry1, Per2, Clock, and Bmal1.

At the level of the organism, the circadian timing system provides internal temporal organization controlled locally by peripheral clocks/oscillators reset by feeding time, and supervised by the master SCN clock mainly reset by ambient light. Impairment of this internal timing due to altered endogenous clockwork or misalignment with external cues, light, and/or mealtime, has a deleterious impact on metabolic health.

Overconsumption of "junk" (most often hypercaloric) food is a well-acknowledged causal factor of obesity. In addition to the type and quantity of food 
eaten, we have reviewed here the importance of the hour of the day when food is eaten. In short, it seems that the recommendation of "doing the right thing at the right time" is relevant for the relation between food intake and circadian rhythmicity, in that significant amounts of ingested fuels at the wrong circadian time (i.e., resting period) can predispose to increased adiposity and other metabolic risks. This link, together with the putative obesogenic property of repeated light-dark shifts or chronic shift work, leads to the concept that we called "chronobesity" ${ }^{65}$ Furthermore, metabolic diseases are associated with circadian disturbances in behavior and physiology, not only in peripheral organs but also in the brain. Together, these findings suggest that management of circadian disorders is especially important in obese and diabetic people to prevent a kind of vicious circle that could amplify health problems.

We propose that dietary strategies for limiting, or preventing, overweight and obesity could be further optimized by taking into account circadian rhythmicity to maintain (or restore) normal temporal organization and synchronization to local time, that is, by developing chronotherapeutic approaches of dieting.

\section{Acknowledgments}

Due to space limitations, we apologize for not citing additional pertinent references. We are grateful to Dr. David Hicks for helpful comments on the manuscript. J.D. is supported by a doctoral fellowship from the French Ministry of National Education and Research, and E.C. is supported by Centre National de Recherche Scientifique, Université de Strasbourg and Agence Nationale pour la Recherche "Jeunes chercheurs/jeunes chercheuses" ANR-07JCJC-0111.

\section{Conflicts of interest}

The authors declare no conflicts of interest.

\section{References}

1. Barness, L.A., J.M. Opitz \& E. Gilbert-Barness. 2007. Obesity: genetic, molecular, and environmental aspects. Am. J. Med. Genet. A 143A: 3016-3034.

2. Tataranni, P.A. \& E. Ravussin. 1997. Effect of fat intake on energy balance. Ann. N.Y. Acad. Sci. 819: 37-43.

3. Welsh, D.K. et al. 1995 . Individual neurons dissociated from rat suprachiasmatic nucleus express independently phased circadian firing rhythms. Neuron. 14: 697-706.
4. Meijer, J.H. \& W.J. Schwartz. 2003. In search of the pathways for light-induced pacemaker resetting in the suprachiasmatic nucleus. J. Biol. Rhythms 18: 235-249.

5. Hattar, S. et al. 2002. Melanopsin-containing retinal ganglion cells: architecture, projections, and intrinsic photosensitivity. Science 295: 1065-1070.

6. Ko, C.H. \& J.S. Takahashi. 2006. Molecular components of the mammalian circadian clock. Hum. Mol. Genet. 15: R271-R277.

7. Preitner, N. et al. 2002. The orphan nuclear receptor REVERBalpha controls circadian transcription within the positive limb of the mammalian circadian oscillator. Cell 110: 251-260.

8. Guillaumond, F. et al. 2005. Differential control of Bmal1 circadian transcription by REV-ERB and ROR nuclear receptors. J. Biol. Rhythms 20: 391-403.

9. Crumbley, C. et al. 2010. Characterization of the core mammalian clock component, NPAS2, as a REVERBalpha/RORalpha target gene. J. Biol. Chem. 285: 35386-35392.

10. Crumbley, C. \& T.P. Burris. 2011. Direct regulation of Clock expression by REV-ERB. PLoS One 6: e17290.

11. Lee, C. et al. 2001. Posttranslational mechanisms regulate the mammalian circadian clock. Cell 107: 855867.

12. Dibner, C., U. Schibler \& U. Albrecht. 2010. The mammalian circadian timing system: organization and coordination of central and peripheral clocks. Annu. Rev. Physiol. 72: 517-549.

13. Watts, A.G. \& L.W. Swanson. 1987. Efferent projections of the suprachiasmatic nucleus: II. Studies using retrograde transport of fluorescent dyes and simultaneous peptide immunohistochemistry in the rat. J. Comp. Neurol. 258: 230-252.

14. la Fleur, S.E. et al. 2000. Polysynaptic neural pathways between the hypothalamus, including the suprachiasmatic nucleus, and the liver. Brain Res. 871: 50-56.

15. Shibata, S. 2004. Neural regulation of the hepatic circadian rhythm. Anat. Rec. A Discov. Mol. Cell. Evol. Biol. 280: 901909.

16. Buijs, R.M. et al. 2001. Parasympathetic and sympathetic control of the pancreas: a role for the suprachiasmatic nucleus and other hypothalamic centers that are involved in the regulation of food intake. J. Comp. Neurol. 431: 405423.

17. Kreier, F. et al. 2002. Selective parasympathetic innervation of subcutaneous and intra-abdominal fat-functional implications. J. Clin. Invest. 110: 1243-1250.

18. Ahima, R.S. \& M.A. Lazar. 2008. Adipokines and the peripheral and neural control of energy balance. Mol. Endocrinol. 22: 1023-1031.

19. Gerozissis, K. 2008. Brain insulin, energy and glucose homeostasis; genes, environment and metabolic pathologies. Eur. J. Pharmacol. 585: 38-49.

20. Nogueiras, R., M.H. Tschop \& J.M. Zigman. 2008. Central nervous system regulation of energy metabolism: ghrelin versus leptin. Ann. N.Y. Acad. Sci. 1126: 14-19.

21. La Fleur, S.E. et al. 1999. A suprachiasmatic nucleus generated rhythm in basal glucose concentrations. J. Neuroendocrinol. 11: 643-652. 
22. Yamamoto, H., K. Nagai \& H. Nakagawa. 1987. Role of SCN in daily rhythms of plasma glucose, FFA, insulin and glucagon. Chronobiol. Int. 4: 483-491.

23. Kalsbeek, A. et al. 2001. The suprachiasmatic nucleus generates the diurnal changes in plasma leptin levels. Endocrinology 142: 2677-2685.

24. Tei, H. et al. 1997. Circadian oscillation of a mammalian homologue of the Drosophila period gene. Nature 389: 512-516.

25. Damiola, F. et al. 2000. Restricted feeding uncouples circadian oscillators in peripheral tissues from the central pacemaker in the suprachiasmatic nucleus. Genes Dev. 14: 2950-2961.

26. Abe, M. et al. 2002. Circadian rhythms in isolated brain regions. J. Neurosci. 22: 350-356.

27. Nagai, K. et al. 1978. Effect of bilateral lesions of the suprachiasmatic nuclei on the circadian rhythm of foodintake. Brain Res. 142: 384-389.

28. Yoo, S.H. et al. 2004. PERIOD2:LUCIFERASE real-time reporting of circadian dynamics reveals persistent circadian oscillations in mouse peripheral tissues. Proc. Natl. Acad. Sci. USA 101: 5339-5346.

29. Akhtar, R.A. et al. 2002. Circadian cycling of the mouse liver transcriptome, as revealed by cDNA microarray, is driven by the suprachiasmatic nucleus. Curr. Biol. 12: 540-550.

30. Hughes, M.E. et al. 2009. Harmonics of circadian gene transcription in mammals. PLoS Genet. 5: e1000442.

31. Miller, B.H. et al. 2007. Circadian and CLOCK-controlled regulation of the mouse transcriptome and cell proliferation. Proc. Natl. Acad. Sci. USA 104: 3342-3347.

32. Oishi, K. et al. 2003. Genome-wide expression analysis of mouse liver reveals CLOCK-regulated circadian output genes. J. Biol. Chem. 278: 41519-41527.

33. Oishi, K. et al. 2005. Genome-wide expression analysis reveals 100 adrenal gland-dependent circadian genes in the mouse liver. DNA Res. 12: 191-202.

34. Ueda, H.R. et al. 2002. A transcription factor response element for gene expression during circadian night. Nature 418: $534-539$.

35. Lowrey, P.L. \& J.S. Takahashi. 2004. Mammalian circadian biology: elucidating genome-wide levels of temporal organization. Annu. Rev. Genomics Hum. Genet. 5: 407-441.

36. Yang, X. et al. 2006. Nuclear receptor expression links the circadian clock to metabolism. Cell 126: 801-810.

37. Reddy, A.B. et al. 2006. Circadian orchestration of the hepatic proteome. Curr. Biol. 16: 1107-1115.

38. Feillet, C.A. et al. 2006. Lack of food anticipation in Per2 mutant mice. Curr. Biol. 16: 2016-2022.

39. Stokkan, K.A. et al. 2001. Entrainment of the circadian clock in the liver by feeding. Science 291: 490-493.

40. Challet, E. 2010. Interactions between light, mealtime and calorie restriction to control daily timing in mammals. J. Comp. Physiol. B 180: 631-644.

41. Unger, J. et al. 1989. Distribution of insulin receptor-like immunoreactivity in the rat forebrain. Neuroscience 31: 143-157.

42. Zigman, J.M. et al. 2006. Expression of ghrelin receptor mRNA in the rat and the mouse brain. J. Comp. Neurol. 494: 528-548.

43. Hakansson, M.L. et al. 1998. Leptin receptor immunoreac- tivity in chemically defined target neurons of the hypothalamus. J. Neurosci. 18: 559-572.

44. Clement, K. et al. 1998. A mutation in the human leptin receptor gene causes obesity and pituitary dysfunction. Nature 392: 398-401.

45. Fukagawa, K. et al. 1992. Advance shift of feeding circadian rhythm induced by obesity progression in Zucker rats. Am. J. Physiol. 263: R1169-R1175.

46. Mistlberger, R.E., H. Lukman \& B.G. Nadeau. 1998. Circadian rhythms in the Zucker obese rat: assessment and intervention. Appetite 30: 255-267.

47. Murakami, D.M., B.A. Horwitz \& C.A. Fuller. 1995. Circadian rhythms of temperature and activity in obese and lean Zucker rats. Am. J. Physiol. 269: R1038-R1043.

48. Sanchez-Alavez, M. et al. 2007. Night eating and obesity in the EP3R-deficient mouse. Proc. Natl. Acad. Sci. USA 104: 3009-3014.

49. Danguir, J. 1989. Sleep patterns in the genetically obese Zucker rat: effect of acarbose treatment. Am. J. Physiol. 256: R281-R283.

50. Megirian, D., J. Dmochowski \& G.A. Farkas. 1998. Mechanism controlling sleep organization of the obese Zucker rats. J. Appl. Physiol. 84: 253-256.

51. Kudo, T. et al. 2004. Night-time restricted feeding normalises clock genes and Pai-1 gene expression in the $\mathrm{db} / \mathrm{db}$ mouse liver. Diabetologia 47: 1425-1436.

52. Laposky, A.D. et al. 2006. Altered sleep regulation in leptindeficient mice. Am. J. Physiol. Regul. Integr. Comp. Physiol. 290: R894-R903.

53. Laposky, A.D. et al. 2008. Sleep-wake regulation is altered in leptin-resistant $(\mathrm{db} / \mathrm{db})$ genetically obese and diabetic mice. Am. J. Physiol. Regul. Integr. Comp. Physiol. 295: R2059-R2066.

54. Sans-Fuentes, M.A., A. Diez-Noguera \& T. Cambras. 2010. Light responses of the circadian system in leptin deficient mice. Physiol. Behav. 99: 487-494.

55. Ando, H. et al. 2011. Impairment of peripheral circadian clocks precedes metabolic abnormalities in ob/ob mice. Endocrinology 152: 1347-1354.

56. Ando, H. et al. 2005. Rhythmic messenger ribonucleic acid expression of clock genes and adipocytokines in mouse visceral adipose tissue. Endocrinology 146: 5631-5636.

57. Kaneko, K. et al. 2009. Obesity alters circadian expressions of molecular clock genes in the brainstem. Brain Res. 1263: $58-68$.

58. West, D.B. \& B. York. 1998. Dietary fat, genetic predisposition, and obesity: lessons from animal models. Am. J. Clin. Nutr. 67: 505S-512S.

59. Hill, J.O. et al. 1992. Development of dietary obesity in rats: influence of amount and composition of dietary fat. Int. J. Obes. Relat. Metab. Disord. 16: 321-333.

60. Cha, M.C., C.J. Chou \& C.N. Boozer. 2000. High-fat diet feeding reduces the diurnal variation of plasma leptin concentration in rats. Metabolism 49: 503-507.

61. Havel, P.J. et al. 1999. High-fat meals reduce 24-h circulating leptin concentrations in women. Diabetes 48: 334 341

62. Kohsaka, A. et al. 2007. High-fat diet disrupts behavioral and molecular circadian rhythms in mice. Cell Metab. 6: 414-421. 
63. Jenkins, J.B. et al. 2006. Sleep is increased in mice with obesity induced by high-fat food. Physiol. Behav. 87: 255262.

64. Wells, A.S. et al. 1997. Influences of fat and carbohydrate on postprandial sleepiness, mood, and hormones. Physiol. Behav. 61: 679-686.

65. Mendoza, J., P. Pevet \& E. Challet. 2008. High-fat feeding alters the clock synchronization to light. J. Physiol. 586: 5901-5910.

66. Garaulet, M. et al. 2011. An approximation to the temporal order in endogenous circadian rhythms of genes implicated in human adipose tissue metabolism. J. Cell Physiol. 226: 2075-2080.

67. Otway, D.T. et al. 2011. Rhythmic diurnal gene expression in human adipose tissue from individuals who are lean, overweight, and type 2 diabetic. Diabetes 60: 1577-1581.

68. Rudic, R.D. et al. 2004. BMAL1 and CLOCK, two essential components of the circadian clock, are involved in glucose homeostasis. PLoS Biol. 2: e377.

69. Barnea, M., Z. Madar \& O. Froy. 2009. High-fat diet delays and fasting advances the circadian expression of adiponectin signaling components in mouse liver. Endocrinology 150: 161-168.

70. Yanagihara, H. et al. 2006. High-fat feeding exerts minimal effects on rhythmic mRNA expression of clock genes in mouse peripheral tissues. Chronobiol. Int. 23: 905-914.

71. Hsieh, M.C. et al. 2010. Abnormal expressions of circadianclock and circadian clock-controlled genes in the livers and kidneys of long-term, high-fat-diet-treated mice. Int. J. Obes. (Lond) 34: 227-239.

72. Turek, F.W. et al. 2005. Obesity and metabolic syndrome in circadian Clock mutant mice. Science 308: 1043-1045.

73. Doi, R., K. Oishi \& N. Ishida. 2010. CLOCK regulates circadian rhythms of hepatic glycogen synthesis through transcriptional activation of Gys2. J. Biol. Chem. 285: 2211422121.

74. Kennaway, D.J. et al. 2007. Metabolic homeostasis in mice with disrupted Clock gene expression in peripheral tissues. Am. J. Physiol. Regul. Integr. Comp. Physiol. 293: R1528R1537.

75. Oishi, K. et al. 2006. Disrupted fat absorption attenuates obesity induced by a high-fat diet in Clock mutant mice. FEBS Lett. 580: 127-130.

76. Kudo, T. et al. 2007. Attenuating effect of clock mutation on triglyceride contents in the ICR mouse liver under a high-fat diet. J. Biol. Rhythms 22: 312-323.

77. Kudo, T. et al. 2008. Clock mutation facilitates accumulation of cholesterol in the liver of mice fed a cholesterol and/or cholic acid diet. Am. J. Physiol. Endocrinol. Metab. 294: E120-130.

78. Oishi, K. et al. 2006. CLOCK is involved in obesity-induced disordered fibrinolysis in ob/ob mice by regulating PAI-1 gene expression. J. Thromb. Haemost. 4: 1774-1780.

79. Shimba, S. et al. 2005. Brain and muscle Arnt-like protein-1 (BMAL1), a component of the molecular clock, regulates adipogenesis. Proc. Natl. Acad. Sci. USA 102: 12071-12076.

80. Lamia, K.A., K.F. Storch \& C.J. Weitz. 2008. Physiological significance of a peripheral tissue circadian clock. Proc. Natl. Acad. Sci. USA 105: 15172-15177.
81. Woon, P.Y. et al. 2007. Aryl hydrocarbon receptor nuclear translocator-like (BMAL1) is associated with susceptibility to hypertension and type 2 diabetes. Proc. Natl. Acad. Sci. USA 104: 14412-14417.

82. Scott, E.M., A.M. Carter \& P.J. Grant. 2008. Association between polymorphisms in the Clock gene, obesity and the metabolic syndrome in man. Int. J. Obes. (Lond) 32: 658-662.

83. Sookoian, S. et al. 2008. Genetic variants of Clock transcription factor are associated with individual susceptibility to obesity. Am. J. Clin. Nutr. 87: 1606-1615.

84. Sadacca, L.A. et al. 2011. An intrinsic circadian clock of the pancreas is required for normal insulin release and glucose homeostasis in mice. Diabetologia 54: 120-124.

85. Marcheva, B. et al. 2010. Disruption of the clock components CLOCK and BMAL1 leads to hypoinsulinaemia and diabetes. Nature 466: 627-631.

86. Yang, S. et al. 2009. The role of mPer2 clock gene in glucocorticoid and feeding rhythms. Endocrinology 150: 21532160 .

87. Grimaldi, B. et al. 2010. PER2 controls lipid metabolism by direct regulation of PPARgamma. Cell Metab. 12: 509-520.

88. Dallmann, R. et al. 2006. Impaired daily glucocorticoid rhythm in Per1 (Brd) mice. J. Comp. Physiol. A 192: 769775 .

89. Dallmann, R. \& D.R. Weaver. 2010. Altered body mass regulation in male mPeriod mutant mice on high-fat diet. Chronobiol. Int. 27: 1317-1328.

90. Raspe, E. et al. 2001. Transcriptional regulation of apolipoprotein C-III gene expression by the orphan nuclear receptor RORalpha. J. Biol. Chem. 276: 2865-2871.

91. Lau, P. et al. 2004. RORalpha regulates the expression of genes involved in lipid homeostasis in skeletal muscle cells: caveolin-3 and CPT-1 are direct targets of ROR. J. Biol. Chem. 279: 36828-36840.

92. Fontaine, C. et al. 2003. The orphan nuclear receptor RevErbalpha is a peroxisome proliferator-activated receptor (PPAR) gamma target gene and promotes PPARgammainduced adipocyte differentiation. J. Biol. Chem. 278: 37672-37680.

93. Wang, J. \& M.A. Lazar. 2008. Bifunctional role of Reverbalpha in adipocyte differentiation. Mol. Cell. Biol. 28: 2213-2220.

94. Le Martelot, G. et al. 2009. REV-ERBalpha participates in circadian SREBP signaling and bile acid homeostasis. PLoS Biol. 7: e1000181.

95. Raspe, E. et al. 2002. Identification of Rev-erbalpha as a physiological repressor of apoC-III gene transcription. J. Lipid Res. 43: 2172-2179.

96. Schmutz, I. et al. 2010. The mammalian clock component PERIOD2 coordinates circadian output by interaction with nuclear receptors. Genes Dev. 24: 345-357.

97. Baggs, J.E. \& C.B. Green. 2003. Nocturnin, a deadenylase in Xenopus laevis retina: a mechanism for posttranscriptional control of circadian-related mRNA. Curr. Biol. 13: 189-198.

98. Green, C.B. et al. 2007. Loss of Nocturnin, a circadian deadenylase, confers resistance to hepatic steatosis and dietinduced obesity. Proc. Natl. Acad. Sci. USA 104: 98889893. 
99. Karatsoreos, I.N. et al. 2011. Disruption of circadian clocks has ramifications for metabolism, brain, and behavior. Proc. Natl. Acad. Sci. USA 108: 1657-1662.

100. Martino, T.A. et al. 2007. Disturbed diurnal rhythm alters gene expression and exacerbates cardiovascular disease with rescue by resynchronization. Hypertension 49: 11041113.

101. Scheer, F.A. et al. 2009. Adverse metabolic and cardiovascular consequences of circadian misalignment. Proc. Natl. Acad. Sci. USA 106: 4453-4458.

102. Fonken, L.K. et al. 2010. Light at night increases body mass by shifting the time of food intake. Proc. Natl. Acad. Sci. USA 107: 18664-18669.

103. Tsai, L.L. et al. 2005. Repeated light-dark shifts speed up body weight gain in male F344 rats. Am. J. Physiol. Endocrinol. Metab. 289: E212-E217.

104. Bartol-Munier, I. et al. 2006. Combined effects of highfat feeding and circadian desynchronization. Int. J. Obes. (Lond) 30: 60-67.

105. Mishra, A. et al. 2009. Proteomic changes in the hypothalamus and retroperitoneal fat from male F344 rats subjected to repeated light-dark shifts. Proteomics 9: 4017-4028.

106. Nakamura, K. et al. 1997. Shift work and risk factors for coronary heart disease in Japanese blue-collar workers: serum lipids and anthropometric characteristics. Occup. Med. (Lond) 47: 142-146.

107. Karlsson, B.H. et al. 2003. Metabolic disturbances in male workers with rotating three-shift work. Results of the WOLF study. Int. Arch. Occup. Environ. Health 76: 424430.

108. Sookoian, S. et al. 2007. Effects of rotating shift work on biomarkers of metabolic syndrome and inflammation. J. Intern. Med. 261: 285-292.

109. Dochi, M. et al. 2009. Shift work is a risk factor for increased total cholesterol level: a 14-year prospective cohort study in 6886 male workers. Occup. Environ. Med. 66: 592-597.

110. Di Lorenzo, L. et al. 2003. Effect of shift work on body mass index: results of a study performed in 319 glucose-tolerant men working in a Southern Italian industry. Int. J. Obes. Relat. Metab. Disord. 27: 1353-1358.

111. Suwazono, Y. et al. 2006. Long-term longitudinal study on the relationship between alternating shift work and the onset of diabetes mellitus in male Japanese workers. J. Occup. Environ. Med. 48: 455-461.

112. Barf, R.P., P. Meerlo \& A.J. Scheurink. 2010. Chronic sleep disturbance impairs glucose homeostasis in rats. Int. J. Endocrinol. 2010: 819414.

113. Spiegel, K. et al. 2009. Effects of poor and short sleep on glucose metabolism and obesity risk. Nat. Rev. Endocrinol. 5: 253-261.

114. Nedeltcheva, A.V. et al. 2009. Exposure to recurrent sleep restriction in the setting of high caloric intake and physical inactivity results in increased insulin resistance and reduced glucose tolerance. J. Clin. Endocrinol. Metab. 94: 3242-3250.

115. Hara, R. et al. 2001. Restricted feeding entrains liver clock without participation of the suprachiasmatic nucleus. Genes Cells 6: 269-278.

116. Sutton, G.M. et al. 2008. The melanocortin-3 receptor is required for entrainment to meal intake. J. Neurosci. 28: 12946-12955.
117. Castillo, M.R. et al. 2004. Entrainment of the master circadian clock by scheduled feeding. Am. J. Physiol. Regul. Integr. Comp. Physiol. 287: R551-R555.

118. Martinez-Merlos, M.T. et al. 2004. Dissociation between adipose tissue signals, behavior and the food-entrained oscillator. J. Endocrinol. 181: 53-63.

119. Arble, D.M. et al. 2009. Circadian timing of food intake contributes to weight gain. Obesity 17: 2100-2102.

120. Salgado-Delgado, R. et al. 2008. Internal desynchronization in a model of night-work by forced activity in rats. Neuroscience 154: 922-931.

121. Salgado-Delgado, R. et al. 2010. Food intake during the normal activity phase prevents obesity and circadian desynchrony in a rat model of night work. Endocrinology 151: 1019-1029.

122. Arendt, J. 2010. Shift work: coping with the biological clock. Occup. Med. (Lond) 60: 10-20.

123. Goel, N. et al. 2009. Circadian rhythm profiles in women with night eating syndrome. J. Biol. Rhythms 24: 85-94.

124. Colles, S.L., J.B. Dixon \& P.E. O’Brien. 2007. Night eating syndrome and nocturnal snacking: association with obesity, binge eating and psychological distress. Int. J. Obes. (Lond) 31: 1722-1730.

125. Lundgren, J.D. et al. 2010. The relationship of night eating to oral health and obesity in community dental clinic patients. Gen. Dent. 58: e134-e139.

126. Striegel-Moore, R.H. et al. 2010. Nocturnal eating: association with binge eating, obesity, and psychological distress. Int. J. Eat. Disord. 43: 520-526.

127. Teboul, M. et al. 2008. The nuclear hormone receptor family round the clock. Mol. Endocrinol. 22: 25732582.

128. Adelmant, G. et al. 1996. A functional Rev-erb alpha responsive element located in the human Rev-erb alpha promoter mediates a repressing activity. Proc. Natl. Acad. Sci. USA 93: 3553-3558.

129. Raspe, E. et al. 2002. Transcriptional regulation of human Rev-erbalpha gene expression by the orphan nuclear receptor retinoic acid-related orphan receptor alpha. J. Biol. Chem. 277: 49275-49281.

130. Vu-Dac, N. et al. 1997. Transcriptional regulation of apolipoprotein A-I gene expression by the nuclear receptor RORalpha. J. Biol. Chem. 272: 22401-22404.

131. Kallen, J. et al. 2004. Crystal structure of the human RORalpha Ligand binding domain in complex with cholesterol sulfate at 2.2 A. J. Biol .Chem. 279: 14033-14038.

132. Vu-Dac, N. et al. 1998. The nuclear receptors peroxisome proliferator-activated receptor alpha and Rev-erbalpha mediate the species-specific regulation of apolipoprotein A-I expression by fibrates. J. Biol. Chem. 273: 2571325720.

133. Yin, L. \& M.A. Lazar. 2005. The orphan nuclear receptor Rev-erbalpha recruits the $\mathrm{N}$-CoR/histone deacetylase 3 corepressor to regulate the circadian Bmall gene. Mol. Endocrinol. 19: 1452-1459.

134. Alenghat, T. et al. 2008. Nuclear receptor corepressor and histone deacetylase 3 govern circadian metabolic physiology. Nature 456: 997-1000.

135. Feng, D. et al. 2011. A circadian rhythm orchestrated by histone deacetylase 3 controls hepatic lipid metabolism. Science 331: 1315-1319. 
136. Tsiftsoglou, A.S., A.I. Tsamadou \& L.C. Papadopoulou. 2006. Heme as key regulator of major mammalian cellular functions: molecular, cellular, and pharmacological aspects. Pharmacol. Ther. 111: 327-345.

137. Kaasik, K. \& C.C. Lee. 2004. Reciprocal regulation of haem biosynthesis and the circadian clock in mammals. Nature 430: 467-471.

138. Raghuram, S. et al. 2007. Identification of heme as the ligand for the orphan nuclear receptors REV-ERBalpha and REV-ERBbeta. Nat. Struct. Mol. Biol. 14: 1207-1213.

139. Zheng, B. et al. 2001. Nonredundant roles of the mPer1 and mPer2 genes in the mammalian circadian clock. Cell 105: 683-694.

140. Handschin, C. et al. 2005. Nutritional regulation of hepatic heme biosynthesis and porphyria through PGC-1alpha. Cell 122: 505-515.

141. Lin, J., C. Handschin \& B.M. Spiegelman. 2005. Metabolic control through the PGC-1 family of transcription coactivators. Cell Metab. 1: 361-370.

142. Liu, C. et al. 2007. Transcriptional coactivator PGC-1alpha integrates the mammalian clock and energy metabolism. Nature 447: 477-481.

143. Dioum, E.M. et al. 2002. NPAS2: a gas-responsive transcription factor. Science 298: 2385-2387.

144. Gervois, P. et al. 2000. Regulation of lipid and lipoprotein metabolism by PPAR activators. Clin. Chem. Lab. Med. 38: 3-11.

145. Yoon, M. 2009. The role of PPARalpha in lipid metabolism and obesity: focusing on the effects of estrogen on PPARalpha actions. Pharmacol. Res. 60: 151-159.

146. Canaple, L. et al. 2006. Reciprocal regulation of brain and muscle Arnt-like protein 1 and peroxisome proliferatoractivated receptor alpha defines a novel positive feedback loop in the rodent liver circadian clock. Mol. Endocrinol. 20: $1715-1727$.

147. Oishi, K., H. Shirai \& N. Ishida. 2005. CLOCK is involved in the circadian transactivation of peroxisome-proliferatoractivated receptor alpha (PPARalpha) in mice. Biochem. J. 386: 575-581.

148. Gervois, P. et al. 1999. Fibrates increase human REVERBalpha expression in liver via a novel peroxisome proliferator-activated receptor response element. Mol. Endocrinol. 13: 400-409.

149. Puigserver, P. et al. 1998. A cold-inducible coactivator of nuclear receptors linked to adaptive thermogenesis. Cell 92: 829-839.

150. Sears, I.B. et al. 1996. Differentiation-dependent expression of the brown adipocyte uncoupling protein gene: regulation by peroxisome proliferator-activated receptor gamma. Mol. Cell. Biol. 16: 3410-3419.

151. Asher, G. \& U. Schibler. 2011. Crosstalk between components of circadian and metabolic cycles in mammals. Cell Metab. 13: 125-137.

152. Rutter, J. et al. 2001. Regulation of clock and NPAS2 DNA binding by the redox state of NAD cofactors. Science 293: 510-514.

153. Williamson, D.H., P. Lund \& H.A. Krebs. 1967. The redox state of free nicotinamide-adenine dinucleotide in the cytoplasm and mitochondria of rat liver. Biochem. J. 103: 514-527.
154. Yu, J. \& J. Auwerx. 2009. The role of sirtuins in the control of metabolic homeostasis. Ann. N.Y. Acad. Sci. 1173(Suppl 1): E10-E19.

155. Dali-Youcef, N. et al. 2007. Sirtuins: the 'magnificent seven', function, metabolism and longevity. Ann. Med. 39: 335345.

156. Blander, G. \& L. Guarente. 2004. The Sir2 family of protein deacetylases. Annu. Rev. Biochem. 73: 417-435.

157. Asher, G. et al. 2008. SIRT1 regulates circadian clock gene expression through PER2 deacetylation. Cell 134: 317328.

158. Nakahata, Y. et al. 2008. The NAD+-dependent deacetylase SIRT1 modulates CLOCK-mediated chromatin remodeling and circadian control. Cell 134: 329-340.

159. Rodgers, J.T. et al. 2005. Nutrient control of glucose homeostasis through a complex of PGC-1alpha and SIRT1. $\mathrm{Na}$ ture 434: 113-118.

160. Purushotham, A. et al. 2009. Hepatocyte-specific deletion of SIRT1 alters fatty acid metabolism and results in hepatic steatosis and inflammation. Cell Metab. 9: 327-338.

161. Picard, F. et al. 2004. Sirt1 promotes fat mobilization in white adipocytes by repressing PPAR-gamma. Nature 429: 771-776.

162. Villaret, A. et al. 2010. Adipose tissue endothelial cells from obese human subjects: differences among depots in angiogenic, metabolic, and inflammatory gene expression and cellular senescence. Diabetes 59: 2755-2763.

163. Barzilai, N. \& I. Gabriely. 2001. The role of fat depletion in the biological benefits of caloric restriction. J. Nutr. 131: 903S-906S.

164. Kahn, B.B. et al. 2005. AMP-activated protein kinase: ancient energy gauge provides clues to modern understanding of metabolism. Cell Metab. 1: 15-25.

165. Canto, C. et al. 2009. AMPK regulates energy expenditure by modulating NAD + metabolism and SIRT1 activity. $\mathrm{Na}$ ture 458: 1056-1060.

166. Vieira, E. et al. 2008. Relationship between AMPK and the transcriptional balance of clock-related genes in skeletal muscle. Am. J. Physiol. Endocrinol. Metab. 295: E1032E1037.

167. Lamia, K.A. et al. 2009. AMPK regulates the circadian clock by cryptochrome phosphorylation and degradation. Science 326: 437-440.

168. Um, J.H. et al. 2007. Activation of $5^{\prime}$-AMP-activated kinase with diabetes drug metformin induces casein kinase Iepsilon (CKIepsilon)-dependent degradation of clock protein mPer2. J. Biol. Chem. 282: 20794-20798.

169. Um, J.H. et al. 2011. AMPK regulates circadian rhythms in a tissue- and isoform-specific manner. PLoS One 6: e18450.

170. Ramsey, K.M. et al. 2009. Circadian clock feedback cycle through NAMPT-mediated NAD+ biosynthesis. Science 324: 651-654.

171. Nakahata, Y. et al. 2009. Circadian control of the NAD+ salvage pathway by CLOCK-SIRT1. Science 324: 654657.

172. Bordone, L. \& L. Guarente. 2005. Calorie restriction, SIRT1 and metabolism: understanding longevity. Nat. Rev. Mol. Cell. Biol. 6: 298-305.

173. O’Neill, J.S. \& A.B. Reddy. 2011. Circadian clocks in human red blood cells. Nature 469: 498-503. 